\title{
Diaģnóstico da Restrição de Crescimento Fetal pela Relação Diâmetro Transverso do Cerebelo e Circunferência Abdominal
}

\author{
Diagnosis of Fetal Growth Restriction by Transverse Cerebellar \\ Diameter/Abdominal Circumference Ratio
}

José de Arimatea Barreto, Carlos Augusto Alencar Júnior

\begin{abstract}
RESUM0
Objetivo: testar a validade da relação diâmetro transverso do cerebelo (DTC)/circunferência abdominal (CA) no diagnóstico da restrição de crescimento fetal (RCF), determinando seu melhor ponto de corte e acurácia nas restrições simétrica e assimétrica.

Métodos: estudo prospectivo, transversal, envolvendo 250 gestantes com gravidez única, idade gestacional entre a $20^{\underline{a}}$ e a $42^{\underline{a}}$ semana, confirmada por ultra-sonografia. A medida do DTC foi obtida colocando-se os calipers nas margens externas do cerebelo, após sua localização na fossa posterior, com suave rotação do transdutor abaixo do plano do tálamo. A circunferência abdominal foi medida na junção das veias porta esquerda e umbilical. $O$ melhor ponto de corte da relação DTC/CA foi obtido pela curva ROC (receiver operator characteristic). Os neonatos cujas relações DTC/CA foram maiores do que o ponto de corte selecionado foram considerados com RCF. Consideraram-se padrão-ouro para o diagnóstico de RCF recémnascidos cujos pesos situaram-se abaixo do percentil 10. Neonatos com RCF e índice ponderal de Rohrer entre 2,2 e 3 foram considerados simétricos e abaixo de 2,2, assimétricos.

Resultados: o ponto de corte da relação DTC/CA, obtido pela curva ROC foi 16,1. A sensibilidade, especificidade, acurácia, valores preditivos positivo e negativo e razões de verossimilhança positiva e negativa foram de 77,4; 82,6;38,7; 96,3; 82; 4,5 e 3,7\%, respectivamente. Na RCF simétrica a sensibilidade e especificidade foram de 80,8 e 81,7\%, respectivamente. Na assimétrica, a sensibilidade e especificidade foram de 60 e $75 \%$, respectivamente.

Conclusão: a relação DTC/CA mostrou-se eficaz no diagnóstico da RCF simétrica e assimétrica.
\end{abstract}

PALAVRAS-CHAVE: Restrição de crescimento fetal. Circunferência abdominal. Diâmetro cerebelar transverso.

\section{Introdução}

A restrição de crescimento fetal (RCF) constitui a segunda causa de mortalidade perinatal,

Maternidade-Escola Assis Chateaubriand, Departamento de Saúde Materno-Infantil, Faculdade de Medicina da Universidade Federal do Ceará Correspondência:

José de Arimatea Barreto

Rua Álvaro Fernandes 44 - Bairro Damas

60420-570 - Fortaleza - Ceará

Fones: (85) 292-3119 9981-3908 - Fax: 281-6000

e-mail: arimateabarreto@uol.com.br sendo a primeira a prematuridade ${ }^{1}$. A incidência de mortalidade no recém-nascido pré-termo é mais elevada se a restrição de crescimento também estiver presente, estando submetido a aumento de duas a dez vezes nas cifras habituais de mortalidade perinatal ${ }^{2}$.

Permanece associada à alta morbidade precoce por anomalias congênitas, asfixia perinatal, circulação fetal persistente, hipotermia, hipoglicemia e policitemia, mesmo com os avanços na assistência neonatal. A longo prazo, a principal preocupação com os neonatos sobreviventes 
é com o seu desenvolvimento neurológico, que está na dependência do tempo, duração e gravidade do dano cerebral ${ }^{2}$.

Uma das dificuldades na avaliação, tratamento e seguimento dos fetos com alteração de crescimento é a imprecisão na sua definição. A mais comumente utilizada é a que considera RCF igual ao pequeno para a idade gestacional; ou seja, um nascituro cujo peso ao nascimento é inferior a um ponto de corte específico para determinada idade gestacional. No entanto, a expressão RCF, idealmente, deveria ser aplicada a casos em que um processo patológico afeta o crescimento fetal normal e resulta em neonato cujo peso é inferior ao seu potencial inerente ${ }^{3}$. Por outro lado, em razão das dificuldades em determinar, na prática, esse potencial, é que nos dias atuais a maioria dos autores ainda prefere utilizar o percentil 10 como limite inferior de normalidade, e fetos com peso abaixo deste valor como tendo sofrido restrição.

Os fatores implicados na etiologia da RCF podem ser classificados em maternos, fetais e placentários, que são os três compartimentos envolvidos com o crescimento do feto ${ }^{4}$. Entretanto, é mais provável que a maioria dos fetos com o comprometimento do potencial de crescimento tenha agressão que afete os três compartimentos em graus variáveis ${ }^{5}$. Em $40 \%$ dos casos de RCF não é possivel identificar a causa ${ }^{6}$.

Atualmente, a RCF tem sido classificada em três grupos: simétrica, assimétrica e mista. A primeira existe quando o feto é proporcionalmente pequeno desde o início da gravidez. Deve-se a lesão no início da gestação, período durante o qual predomina hiperplasia, afetando o número de células. Infecções intra-uterinas (por exemplo, citomegalovírus, rubéola, toxoplasmose), cromossomopatias, anormalidades estruturais e síndromes genéticas são relatadas como causas. Responde por aproximadamente um terço dos casos. Na RCF assimétrica, o agravo ocorre a partir do final do segundo trimestre. Este é o período em que predomina hipertrofia celular. Relaciona-se com diminuição do crescimento abdominal em relação ao cefálico, com efeitos variáveis sobre o comprimento do fêmur. Insuficiência placentária com diminuição de provisão de nutrientes leva à utilização de glicogênio hepático, diminuição do fígado fetal e redução da circunferência abdominal. O fluxo sangüíneo preferencial para a cabeça fetal dá continuidade ao crescimento deste compartimento, em detrimento dos outros órgãos ${ }^{7}$.

Esses dois tipos de anormalidades de crescimento, muitas vezes, fundem-se, particularmente após privação prolongada da nutrição fetal, afe- tando as fases de hiperplasia e hipertrofia celulares. É o tipo misto de $\mathrm{RCF}^{8}$.

A ultra-sonografia é considerada o instrumento de melhor acurácia no diagnóstico de $\mathrm{RCF}^{7}$, sendo utilizada com esta finalidade há mais de três décadas ${ }^{9}$. É o principal método de confirmação de idade gestacional (dado de informação essencial na determinação da distribuição da biometria) e crescimento fetal ${ }^{10}$. Muitos parâmetros ultra-sonográficos são utilizados no diagnóstico de RCF, tais como diâmetro biparietal, circunferência cefálica (CC), circunferência $a b-$ dominal, comprimento do fêmur, diâmetro transverso do cerebelo (DTC) e estimativa do peso fetal. Razões biométricas (circunferência cefálica/circunferência abdominal, comprimento do fêmur/ circunferência abdominal), medidas de líquido amniótico, estimativa da velocidade de crescimento fetal e dopplervelocimetria têm sido acrescidas ao arsenal diagnóstico de $\mathrm{RCF}^{7}$. Os parâmetros independentes da idade gestacional, como a relação comprimento do fêmur/circunferência abdominal e a razão diâmetro transverso do cerebelo/ circunferência abdominal (DTC/CA), têm sido empregados. Este último é considerado por alguns autores como parâmetro de eleição no diagnóstico de $\mathrm{RCF}^{11-13}$.

O cerebelo fetal pode ser visualizado pela ultra-sonografia na fossa posterior do crânio a partir do final do primeiro trimestre de gestação. A melhor identificação de seus bordos ocorre a partir do segundo trimestre até a $35^{\mathrm{a}}$ semana de gestação. Em fetos com idade gestacional próxima ao termo, a visualização torna-se dificultada. Isto ocorre em virtude da insinuação do pólo cefálico, da diminuição do líquido amniótico, da posição occípito-posterior e do contato da musculatura uterina com a cabeça fetal ${ }^{14}$. Uma forma de atenuar estas dificuldades é fazer a medida do cerebelo em plano coronal ${ }^{15}$.

O DTC é minimamente afetado pelo crescimento fetal alterado ${ }^{16}$. A justificativa para isto seria a manutenção de fluxo sangüíneo adequado para o cerebelo em fetos com asfixia, enquanto o fluxo sangüíneo cortical encontrar-se-ia prejudicado. Isto é sugerido no estudo de Behrman et al. ${ }^{17}$, que conseguiram demonstrar diferença de fluxo sangüíneo entre o córtex e outras áreas filogeneticamente mais antigas, injetando microesferas marcadas com escândio-46, estrôncio-85 e iodo-125 na circulação sangüínea de primatas.

Estudo prospectivo pioneiro da razão DTC/ CA em 162 pacientes sem complicações demonstrou tratar-se de relação independente da idade gestacional, obtendo média de $13,7 \%$, percentil 
cinco de $11,9 \%$ e percentil 95 de 15,9\%, com idade gestacional de 15 a 38 semanas $^{18}$. Em gestações normais, esta relação mostra-se constante a partir do início do segundo até o final do terceiro trimestre $^{12}$.

Como a CA diminui em fetos com RCF, mas o DTC não se altera, a relação DTC/CA eleva-se ${ }^{12}$. O ponto de corte desta elevação, considerado significativo para diagnóstico de RCF, varia entre os diversos autores, desde $15,4^{12}$ até $15,9^{19}$, o primeiro valor apresentando melhor sensibilidade $(73,26 \%)$, especificidade $(80,25 \%)$ e valor preditivo positivo $(79,75 \%)$ e negativo $(73,86 \%)$.

A determinação do melhor ponto de corte é possível pela construção de curva ROC (receiver operator characteristic). Esta curva foi primariamente desenvolvida nos anos 1950 para avaliar a detecção de sinais de radar (daí a sua denominação), não obstante só recentemente tenha se tornado comum o seu uso na área médica. Sua construção é feita colocando-se os valores da sensibilidade (proporção de verdadeiros positivos) nas ordenadas (eixo Y) e o complemento da especificidade ( 1 - especificidade), ou seja, a proporção de falsos positivos nas abscissas (eixo $\mathrm{X}$ ) para diferentes pontos de corte ${ }^{20}$.

Os objetivos deste estudo foram testar a validade da relação DTC/CA como método ultrasonográfico no diagnóstico da RCF, determinando seu melhor ponto de corte pela curva ROC, e verificar sua acurácia nas restrições de crescimento simétrica e assimétrica.

\section{Pacientes e Métodos}

Estudo de teste diagnóstico ultrasonográfico, prospectivo, transversal, conduzido na Maternidade-Escola Assis Chateaubriand (MEAC) da Universidade Federal do Ceará, Fortaleza-Ceará. Foram recrutadas pacientes gestantes atendidas no Ambulatório de Medicina Materno-Fetal (gravidez de alto risco) e as internadas na enfermaria de intercorrências clínico-obstétricas, selecionando-se todas aquelas que atendessem aos critérios de inclusão, no período de 18 de dezembro de 2001 a 13 de fevereiro de 2003.

Os critérios de inclusão foram: data da última menstruação conhecida, ou ultra-sonografia até a $12^{\text {a }}$ semana que demonstrasse a idade; ultrasonografia precoce (anterior à $22^{\mathrm{a}}$ semana de gestação) que demonstrasse a idade gestacional em concordância com a idade menstrual, admitindose discordância de até uma semana; idade gestacional, no momento de inclusão na pesqui- sa, de 20 semanas ou mais; gravidez única e feto vivo. Foram considerados critérios de exclusão: anomalias fetais do sistema nervoso central, do abdome ou de ambos; morte fetal; parto após 14 dias da última ultra-sonografia, sendo os dados deste exame os que foram utilizados para análise.

Foram pesquisadas as seguintes variáveis: diâmetro biparietal, circunferência cefálica, diâmetro transverso do cerebelo, circunferência abdominal, comprimento do fêmur, peso fetal, circunferência cefálica/circunferência abdominal, comprimento do fêmur/circunferência abdominal, DTC/CA) e idade gestacional. Em relação ao neonato, foram pesquisados sexo, peso, comprimento e índice ponderal de Rohrer.

Todas as ultra-sonografias foram realizadas pelo próprio pesquisador (JAB), usando aparelho da marca Siemens, modelo Sonoline Versa-Pro, equipado com escala de cinza, em tempo real, com sonda abdominal convexa de 3,5 $\mathrm{MHz}$ e transvaginal de $6,5 \mathrm{MHz}$ de freqüência.

Com exceção do peso, que foi estimado em gramas, todas as outras medidas fetais foram obtidas em milímetros, com utilização de caliper eletrônico multidirecional. Cada medida foi aferida três vezes, sendo considerada para análise a medida resultante da média aritmética, seguindo as normas de arredondamento, quando necessário.

O DTC foi medido pela técnica de Goldstein et al. ${ }^{11}$ : localização do cerebelo na fossa posterior por meio de rotação do transdutor de aproximadamente $30^{\circ}$ a partir do plano que identifica os tálamos, cavum do septo pelúcido, terceiro ventrículo e cisterna magna, colocando-se os calipers nas margens externas dos hemisférios cerebelares.

A CA foi medida a partir de corte transversal no nível da junção da veia umbilical com a veia porta esquerda. Foi obtida por meio de perímetro traçado ao longo da margem externa do abdome fetal $^{18}$. O peso fetal foi estimado pela técnica de Hadlock et al. ${ }^{21}$. A relação DTC/CA foi calculada pela divisão do DTC pela CA e em seguida multiplicando-se por $100^{18}$. No neonato, foram avaliados peso e comprimento logo após o nascimento, além do índice ponderal de Rohrer, estimado pela equação: IP $=\mathrm{P} / \mathrm{E}^{3} \times 100$. Nesta fórmula, IP é o índice ponderal de Rohrer, $\mathrm{P}$ é o peso ao nascimento expresso em gramas e $\mathrm{E}$, a estatura do recém-nascido em centímetros ${ }^{3,22,23}$.

Foram classificados como recém-nascidos que sofreram RCF todos aqueles pequenos para a idade gestacional, ou seja, que se encontraram com peso abaixo do percentil 10 para a idade gestacional das curvas de Lubchenco corrigidas para o sexo ${ }^{24}$. 
Consideraram-se com RCF simétrica os recém-nascidos com pesos situados abaixo do percentil 10 para a idade gestacional das curvas de Lubchenco corrigidas para o sexo ${ }^{24}$, com índice ponderal situado entre 2,20 e 3,0, e assimétrica, aqueles abaixo de $2,2^{3,24}$.

As análises foram feitas com o software de estatística Statistical Package for the Social Sciences (SPSS) 10.0 for Windows, empregando-se os seguintes recursos: análise descritiva das variáveis quantitativas em função da restrição de crescimento, calculando, média, desvio padrão (DP), mínimo, mediana e máximo; teste de Mann-Whitney para a hipótese de igualdade de médias; construção de curva ROC para determinar o melhor ponto de corte da relação DTC/CA (teste diagnóstico) capaz de identificar RCF; determinação, de acordo com o ponto de corte escolhido, das propriedades do método diagnóstico, tais como sensibilidade, especificidade, acurácia, valores preditivos positivo e negativo, razões de verossimilhanças para os testes positivos e negativos e incidência; análise do teste DTC/CA na dependência do tipo de $\mathrm{RCF}$ (simétrica ou assimétrica) pelo teste de McNemar (igualdade de proporções para dados pareados).

O presente estudo foi aprovado pelo Comitê de Ética em Pesquisa do Complexo Hospitalar da Universidade Federal do Ceará - COMEPE, conforme ofício no 294/2001, protocolo no 204/01, atendendo a todas as suas exigências.

\section{Resultados}

Das 290 pacientes elegiveis para o estudo, duas foram excluídas antes do parto, uma em virtude do diagnóstico de hidrocefalia durante o seguimento ultra-sonográfico e outra por óbito fetal. Trinta e oito tiveram o parto após 14 dias do último exame ultra-sonográfico ou não tiveram acompanhamento pós-parto para coleta dos dados dos recém-nascidos, totalizando quarenta perdas. Permaneceram na pesquisa 250 pacientes para análise final, tendo sido efetuadas 551 ultrasonografias. O parto ocorreu com idade gestacional entre a $25^{\mathrm{a}}$ e a $42^{\mathrm{a}}$ semanas completas.

A idade gestacional na data do parto, no grupo com RCF, variou de 25 a 42 semanas completas, média de $36,5(\mathrm{DP}=4,6)$. No grupo sem restrição, variou de 27 a 42, média de 38,8 (DP=2,3). O grupo com RCF mostrou média de nascimento 2,3 semanas inferior ao grupo sem restrição $(p=0,0023)$, portanto, estatisticamente significante (Tabela 1).
Tabela 1 - Idade gestacional em função da restrição de crescimento fetal.

\begin{tabular}{lccccccc}
\hline \multicolumn{7}{c}{ Idade gestacional } \\
& Média & Mínima Mediana Máxima & DP & $\mathbf{p}^{*}$ \\
\hline $\begin{array}{l}\text { Neonatos com } \\
\text { RCF }(n=31)\end{array}$ & 36,5 & 25 & 37 & 42 & 4,6 & 0,0023 \\
Neonatos sem & & & & & & \\
RCF $(n=219)$ & 38,8 & 27 & 39 & 42 & 2,3 & 0,0023 \\
\hline
\end{tabular}

Idade gestacional $=$ idade gestacional na data do parto em semanas completas; $\mathrm{RCF}=$ restrição de crescimento fetal; $n=$ número de neonatos; $D P=$ desvio padrão; $p=$ nível de significância estatística pelo teste de Mann-Whitney.

Dentre os recém-nascidos com RCF (total de $31)$, dez $(32,26 \%)$ eram prematuros, 18 (58,06\%) de termo e três $(9,68 \%)$ pós-termo. No grupo sem RCF (total de 219), 24 (10,96\%) eram prematuros, $184(84,02 \%)$ de termo e 11 (5,02\%) pós-termo.

O ponto de corte $\geq 16,1$ obtido pela análise da curva ROC (Figura 1) mostra o melhor contrabalanço entre sensibilidade e especificidade da relação DTC/CA para o diagnóstico de RCF. Corresponde ao ponto da curva ROC mais próximo ao canto superior esquerdo do gráfico. Fornece sensibilidade de $77,4 \%$ (erro padrão de $7,5 \%$, intervalo de confiança de 62,6 a $92,1 \%$ ) e especificidade de $82,6 \%$ (erro padrão de $2,5 \%$, intervalo de confiança de 77,5 a $87,6 \%)$. A taxa de resultados falso- positivos nesse ponto é de $17,4 \%$ (1- especificidade).

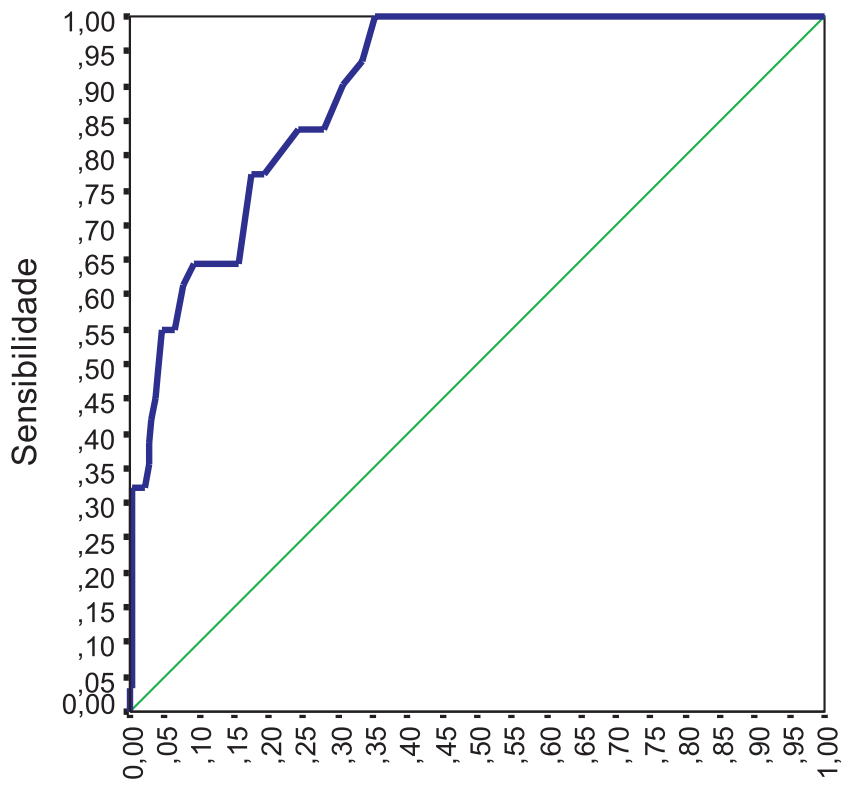

\section{1 - Especificidade}

Figura 1 - Curva ROC (receiver operator characteristic) para determinar o ponto de corte de melhor contrabalanço entre sensibilidade e especificidade da relação diâmetro transverso do cerebelo/circunferência abdominal no diagnóstico de restrição de crescimento fetal. Área sob a curva ROC $=0,897$. Intervalos de confiança de $95 \%$ para sensibilidade em cada ponto de corte. A diagonal representa teste que não teria nenhuma utilidade diagnóstica. 
A área sob a curva ROC (Figura 1) para diagnóstico de RCF foi de 0,897 e mostra o poder discriminatório do teste, ou seja, sua acurácia global, que corresponde a 89,7\%. Quanto mais próxima de $100 \%$ for a área, maior o poder discriminatório do teste. A área sob a curva ROC foi, portanto, significantemente maior do que 50\% (área sob a curva ROC para um teste sem utilidade diagnóstica).

O teste DTC/CA foi positivo em 62 casos, num total de 250 casos pesquisados. Em 31 recém-nascidos com RCF, o teste diagnosticou 24 casos (verdadeiros positivos). Em 219 recém-nascidos sem RCF, mostrou-se positivo em 38 casos (falso-positivos). Quando o teste foi negativo (188 casos), sete casos apresentaram RCF (falso-negativos) e 181 casos não apresentaram RCF (verdadeiros negativos).

Considerando-se o teste positivo com DTC/ $\mathrm{CA} \geq 16,15$, obteve-se prevalência de neonatos com restrição de crescimento de $12,4 \%$, sensibilidade de $77,4 \%$, especificidade de $82,6 \%$, valores preditivos positivo e negativo de 38,7 e $96,3 \%$, respectivamente, acurácia de $82 \%$ e razões de verossimilhanças positiva e negativa de 4,5 e 3,7.

O teste DTC/CA foi positivo para RCF simétrica em 21 casos (verdadeiros positivos), num total de 26 onde realmente estava presente. Foi positivo em 41 casos de onde de fato a RCF estava ausente (falso-positivos). O teste revelou-se negativo em cinco casos onde a RCF simétrica estava presente (falso-negativos) e em 183 casos de onde ela estava ausente (verdadeiros negativos).

Para RCF assimétrica, o teste DTC/CA foi positivo em três casos (verdadeiros positivos), num total de cinco, onde a restrição estava de fato presente. Em 59 casos positivos ela estava ausente (falso-positivos). O teste mostrou-se negativo em dois casos onde a RCF assimétrica estava presente (falso-negativos) e em 186 casos em que estava ausente (verdadeiros negativos) (Tabela 2).

Tabela 2 - Distribuição dos pacientes segundo a classificação de restrição de crescimento fetal em simétrica e assimétrica e o diagnóstico pelo teste DTC/CA.

\begin{tabular}{lrrrrrr}
\hline $\begin{array}{l}\text { Teste } \\
\text { DTC/CA } \geq 16,15\end{array}$ & \multicolumn{7}{c}{$\begin{array}{c}\text { Restrição de crescimento } \\
\text { fetal (padrão-ouro) }\end{array}$} \\
& Simétrica & Ausente & Total & Assimétrica & Ausente & Total \\
\hline Positivo & 21 & 41 & 62 & 3 & 59 & 62 \\
Negativo & 5 & 183 & 188 & 2 & 186 & 188 \\
Total & 26 & 224 & 250 & 5 & 245 & 250 \\
\hline
\end{tabular}

DTC/CA = diâmetro transverso do cerebelo/circunferência abdominal. Classificação nos tipos simétrica e assimétrica com base no índice ponderal de Rohrer do recém-nascido.

Comparando-se o teste DTC/CA no diagnóstico de RCF simétrica e assimétrica observam-se prevalências de 10,6 e 2,2\%, sensibilidade de 80,8 e
$60 \%$ ( $\mathrm{p}=0,5622)$ especificidade de $82,6 \%$ para ambos. No diagnóstico de RCF simétrica e assimétrica, a análise do teste DTC/CA não mostrou diferença estatisticamente significante $(\mathrm{p}>0,05)$ (Tabela 3).

Tabela 3 - Análise do teste DTC/CA para restrição de crescimento fetal simétrica e assimétrica.

\begin{tabular}{lccc}
\hline Teste DTC/CA $\geq 16,15$ & RCF Simétrica & RCF Assimétrica & p \\
\hline Prevalência & $10,4 \%$ & $2,0 \%$ & \\
Sensibilidade & $80,8 \%$ & $60,0 \%$ & $>0,05$ \\
Especificidade & $81,7 \%$ & $75,9 \%$ & $>0,05$ \\
Valor preditivo positivo (VPP) & $33,9 \%$ & $4,8 \%$ & \\
Valor preditivo negativo (VPN) & $97,3 \%$ & $98,9 \%$ & \\
Acurácia & $81,6 \%$ & $75,6 \%$ & \\
Razão de verossimilhança & & & \\
$\quad$ positiva (RV +) & 4,4 & 2,5 & \\
Razão de verossimilhança & & & \\
negativa (RV -) & 4,2 & 1,9 & \\
\hline
\end{tabular}

$\mathrm{DTC} / \mathrm{CA}=$ diâmetro transverso do cerebelo/circunferência abdominal; RCF = restrição de crescimento fetal. Classificação nos tipos simétrica e assimétrica com base no índice ponderal de Rohrer do recém-nascido; $p$ = nível de significância (teste de McNemar).

\section{Discussão}

Neste estudo, testou-se a validade da relação DTC/CA como meio diagnóstico ultrasonográfico de restrição de crescimento fetal em amostra com baixa prevalência. Este diagnóstico reveste-se de grande importância pela associação desta entidade com altos índices de morbidade e mortalidade perinatais ${ }^{1,3}$.

Pelo aspecto prático e pela reprodutibilidade na aferição do peso fetal, a maioria dos autores, mesmo sabendo que até $40 \%$ dos neonatos com peso abaixo do percentil 10 para a idade gestacional não sofreram restrição de crescimento, sendo, apenas, constitucionalmente pequenos, ainda considera, nas suas pesquisas, os fetos/neonatos de peso abaixo do percentil 10 como conceito de RCF. Por estes mesmos motivos, preferiu-se, neste estudo, esta definição, que também é a utilizada no serviço onde foi realizada a pesquisa.

A amostra deste estudo é composta de pacientes do ambulatório e da enfermaria de gestação de alto risco em geral (não especificamente de risco para RCF), não se havendo encontrado, na literatura consultada, estudo com esta característica de amostra. Foram analisadas variáveis ultra-sonográficas fetais e neonatais de 250 pacientes, diagnosticando-se 31 casos de recém-nascidos que sofreram RCF, indicando prevalência de $12,4 \%$. Estudo em pacientes ambulatoriais, não especificadas se de baixo ou alto risco para RCF, 
mostrou prevalência de $13,5 \%{ }^{14}$, o que mais se assemelhou a este estudo.

Os primeiros a estabelecer em pontos de corte da relação DTC/CA para o diagnóstico de RCF foram Campbell et al. ${ }^{19}$ e Haller et al. ${ }^{14}$. Posteriormente, Tongsong et al. ${ }^{12}$ foram os pioneiros a idealizar curva ROC para determinar o ponto de corte com o melhor contrabalanço entre sensibilidade e especificidade no diagnóstico de RCF.

Conforme as fontes consultadas, este estudo é o segundo a propor curva ROC, acrescentando a peculiaridade de aplicabilidade do teste no diagnóstico de RCF simétrica e assimétrica.

Tongsong et al. ${ }^{12}$ avaliaram DTC/CA em 167 pacientes com suspeita clínica de RCF e construíram curva ROC. Definiram RCF como o peso abaixo do percentil 10 para a idade gestacional, com base em curva de sua própria população. Constataram prevalência de $51,5 \%$. Seu ponto de corte indicado foi 15,4, obtendo sensibilidade, especificidades, valores preditivos positivo e negativo de 73,26 ; 80,$25 ; 79,75$ e $73,86 \%$, respectivamente.

No presente estudo, o ponto de corte DTC/ CA obtido a partir de curva ROC foi de 16,15. Comparando este estudo com o de Tongsong et al. ${ }^{12}$, que, embora se tratando de amostra com alta prevalência de RCF, é o único com metodologia de curva ROC, verifica-se no presente ensaio que há sensibilidade e especificidade maiores, apesar do ponto de corte maior, quando era de se esperar menor sensibilidade. Em relação ao valor preditivo positivo, o presente estudo apresentou resultado inferior, o que se explica pela prevalência também inferior, já que esta análise é dependente da prevalência.

Campbell et al. ${ }^{19}$ chamam atenção para os casos de RCF, nos quais a relação DTC/CA se manteve normal (29,2\%). Deste percentual, 57\% eram constituídos por RCF grave. Deduzem que, nestes casos, ocorreu também algum grau de diminuição do DTC. Meyer et al. ${ }^{10}$, Haller et al. ${ }^{14}$, Tongsong et al. ${ }^{12}$ e Vinkesteijn et al. ${ }^{13}$ encontraram resultados semelhantes. Neste estudo, os casos graves de restrição também apresentaram DTC/CA normais, principalmente nos casos com prematuridade.

Achado surpreendente deste estudo referese à proporção entre neonatos com restrição de crescimento simétrica e assimétrica, bastante discordante daquela classicamente descrita, de um terço e dois terços, respectivamente. Dos 31 casos com RCF, 26 (83,87\%) foram do tipo simétrica e cinco $(16,13 \%)$ do tipo assimétrica. Atualmente, a proporcionalidade e desproporcionalidade fetais são questionadas. Em algumas populações, a restrição simétrica é encontrada mais freqüentemente do que a assimétrica ${ }^{25}$.
Objetivando neste estudo avaliar a validade da relação DTC/CA no diagnóstico da RCF simétrica e assimétrica, considerando o ponto de corte $\geq 16,1$, observamos que o baixo valor preditivo positivo do teste é conseqüência da baixa prevalência, principalmente no grupo da RCF assimétrica $(2,0 \%)$. Pesquisando nos bancos de dados MEDLINE e LILACS, concluímos que este trabalho é o primeiro que compara a capacidade diagnóstica da relação DTC/CA para os subtipos simétricos e assimétricos de RCF, baseando-se em ponto de corte construído por análise de curva ROC. A relação mostra-se eficiente em ambos os tipos e, acredita-se, com ampla possibilidade de aplicação clínica. Meyer et al. ${ }^{10}$ testaram a acurácia do DTC/CA no diagnóstico dos tipos simétricos e assimétricos sem utilizar curva ROC, encontrando sensibilidade de 98\% no assimétrico e de $71 \%$ no simétrico.

O presente trabalho pretende trazer uma contribuição para o diagnóstico da RCF, somandose às outras formas de diagnóstico. No meio brasileiro, em que percentual elevado de grávidas desconhece a data da menstruação, tem ciclos irregulares ou não fez ultra-sonografia precoce, dificultando a determinação da idade gestacional, reveste-se de grande importância, por tratar-se de método independente da idade gestacional.

Desta forma, sugere-se acrescentar a relação DTC/CA no ponto de corte determinado $(\geq 16,15)$ à prática clínica diária, em populações semelhantes à estudada, sejam elas de risco ou não para RCF. No entanto, não se recomenda o método de forma isolada.

\section{ABSTRACT}

Purpose: to evaluate the validity of transverse cerebellar diameter (TCD)/abdominal circumference (AC) ratio in the diagnosis of fetal growth restriction (FGR), determining its best cutoff value and accuracy in symmetric and asymmetric FGR. Method: a prospective cross-sectional study, carried out in 250 pregnant women with singleton pregnancies, gestational age between 20 and 42 weeks, with ultrasound confirmation. The TCD measurement was obtained by placing the calipers at the outer margins of the cerebellum, after its localization in the posterior fossa, and slightly rotating the transducer below the plane of the thalami. The abdominal circumference was measured at the on junction of the left portal and umbilical veins. The best TCD/AC cutoff ratio was established by the receiver operator characteristic (ROC) curve. Neonates with TCD/AC ratio greater than the cutoff value were diagnosed as having FGR. We classified as gold standard for FGR the newborn infants who presented birth weight below the $10^{\text {th }}$ percentile. Neonates showing FGR and Rohrer ponderal index between 2.2 and 3 were labeled as symmetric and below 2.2, asymmetric. 
Results: the cutoff value calculated by the ROC curve for TCD/ $A C$ ratio was 16.15. The sensitivity, specificity, accuracy, positive and negative predictive values, and likelihood ratio for positive and negative tests were 77.4, 82.6, 38.7, 96.3, 82, 4.5 and 3.7\%, respectively. In the symmetric $F G R$, sensitivity and specificity were 80.8 and $81.7 \%$, respectively. In the asymmetric FGR, sensitivity and specificity were 60 and $75 \%$, respectively. Conclusion: TCD/AC ratio is an effective method in symmetric and asymmetric FGR diagnosis.

KEYWORDS: Fetal growth restriction. Fetal ultrasonography. Transverse cerebellar diameter. Abdominal circumference.

\section{Referências}

1. Resnik R. Intrauterine growth restriction. Obstet Gynecol 2002; 99:490-6.

2. Teberg AJ, Walther FJ, Pena IC. Mortality, morbidity and outcome of the small-for-gestational-age infant. Semin Perinatol 1988;12:84-94.

3. Ott WJ. Diagnosis of intrauterine growth restriction: comparison of ultrasound parameters. Am J Perinatol 2002; 19:133-7.

4. Carrera JM. Crecimiento intrauterino retardado: concepto y frecuencia. In: Carrera JM et al., editores. Crecimiento Fetal Normal y Patológico. $1^{\text {a }}$ ed. Barcelona: Masson; 1997. p. 219-24.

5. Manning FA. Retardo do crescimento intra-uterino. In: Manning FA, editor. Medicina Fetal: perfil biofísico, princípios e aplicabilidade clínica. $1^{\text {a }}$ ed. Rio de Janeiro: Revinter; 2000. p. 305-90.

6. Wollmann HA. Intrauterine growth restriction: definition and etiology. Horm Res 1998; 49 (Suppl 2):1-6.

7. Campbell BA. Utilizing sonography to follow fetal growth. Obstet Gynecol Clin North Am 1998; 25:597-607.

8. Creasy RK, Resnik R. Intrauterine growth restriction. In: Creasy RK, Resnik R, editors. Maternal-Fetal Medicine. $4^{\text {th }}$ ed. Philadelphia: Saunders; 1999. p. 569-84.

9. Almeida PJ, Sá RAM, Lorenzatto MA, Amin Júnior, Netto HC. Medida ultra-sonográfica do diâmetro transverso do cerebelo fetal em gestações normais. J Bras Ginecol 1998; 108:383-93.

10. Meyer WJ, Gauthier D, Ramakrishnan V, Sipos J. Ultrasonographic detection of abnormal fetal growth with the gestational age-independent, transverse cerebellar diameter/abdominal circumference ratio. Am J Obstet Gynecol 1994; 171:1057-63.

11.Goldstein I, Reece EA, Pilu G, Bovicelli L, Hobbins JC. Cerebellar measurements with ultrasonography in the evaluation of fetal growth and development. Am J Obstet Gynecol 1987; 156:1065-9.

12.Tongsong $\mathrm{T}$, Wanapirak $\mathrm{C}$, Thongpadungroj $\mathrm{T}$. Sonographic diagnosis of intrauterine growth restriction (IUGR) by fetal transverse cerebellar di- ameter (TCD)/abdominal circumference (AC) ratio. Int J Gynaecol Obstet 1999; 66:1-5.

13.Vinkesteijn AS, Mulder PG, Wladimiroff JW. Fetal transverse cerebellar diameter measurements in normal and reduced fetal growth. Ultrasound Obstet Gynecol 2000; 15:47-51.

14. Haller H, Petrovic O, Rukavina B. Fetal transverse cerebellar diameter/abdominal circumference ratio in assessing fetal size. Int J Gynaecol Obstet 1995; 50:159-63.

15.Kofinas AD, Simon NV, King K, Clay D, Deardorf J. Comparison of fetal cerebellar measurements by two different techniques. J Ultrasound Med 1992; 11:257-9.

16.Lee W, Barton S, Comstock CH, Bajorek S, Batton D, Kirk JS. Transverse cerebellar diameter: a useful predictor of gestational age for fetuses with asymmetric growth retardation. Am J Obstet Gynecol 1991; 165:1044-50.

17.Behrman RE, Lees MH, Peterson EN, De Lannoy CW, Seeds AE. Distribution of the circulation in the normal and asphyxiated fetal primate. Am J Obstet Gynecol 1970; 108:956-69.

18.Campbell WA, Nardi DA, Vintzileos AM, Rodis JF, Turner GW, Egan JF. Transverse cerebellar diameter/abdominal circumference ratio throughout pregnancy: a gestational age-independent method to assess fetal growth. Obstet Gynecol 1991; 77:893-6.

19. Campbell WA, Vintzileos AM, Rodis JF, Turner GW, Egan JF, Nardi DA. Use of the transverse cerebellar diameter/abdominal circumference ratio in pregnancies at risk for intrauterine growth retardation. J Clin Ultrasound 1994; 22:497-502.

20. Medronho RA, Perez MA. Testes diagnósticos. In: Medronho RA, editor. Epidemiologia. São Paulo: Atheneu; 2002. p. 259-70.

21.Hadlock FP, Harrist RB, Sharman RS, Deter RL, Park SK. Estimation of fetal weight with the use of head, body, and femur measurements - a prospective study. Am J Obstet Gynecol 1985; 151:333-7.

22.Walther FJ, Ramaekers LH. The ponderal index as a measure of the nutritional status at birth and its relation to some aspects of neonatal morbidity. J Perinat Med 1982; 10:42-7.

23.Rahimian J, Varner MW. Disproportionate fetal growth. In: DeCherney AH, Nathan L, editors. Current Obstetric and Gynecological Diagnosis \& Treatment. $9^{\text {th }}$ ed. New York: McGraw-Hill; 2003. p. 301-14.

24.Lubchenco LO, Hansman C, Dressler M, Boyd E. Intrauterine growth as estimated from liveborn birth-weight data at 24 to 42 weeks of gestation. Pediatrics 1963; 32:793-800.

25.Vik T, Markestad T, Ahlsten G, et al. Body proportions and early neonatal morbidity in small-for-gestational-age infants of successive births. Acta Obstet Gynecol Scand Suppl 1997; 165:76-81.

Recebido em: 28/6/2004 Aceito com modificações em: 29/7/2004 